\title{
Towards a Practical Alarm Correlation System
}

\author{
K. Houck, S. Calo, A. Finkel
}

IBM T. J. Watson Research Center

P.O. Box 704

Yorktown Heights, NY 10598

Email: houck@watson.ibm.com

\begin{abstract}
A single fault in a telecommunication network frequently results in a number of alarms being reported to the network operator. This multitude of alarms can easily obscure the real cause of the fault. In addition, when multiple faults occur at approximately the same time, it can be difficult to determine how many faults have occurred, thus creating the possibility that some may be missed. A variety of solution approaches have been proposed in the literature, however, practically deployable, commercial solutions remain elusive. The experiences of the Network Fault and Alarm Correlator and Tester (NetFACT) project, carried out at IBM Research and described in this paper, provide some insight as to why this is the case, and what must be done to overcome the barriers encountered. Our observations are based on experimental use of the NetFACT system to process a live, continuous alarm stream from a portion of the Advantis physical backbone network, one of the largest private telecommunications networks in the world.

The NetFACT software processes the incoming alarm stream and determines the faults from the alarms. It attempts to narrow down the likely root causes of each fault, to the greatest extent possible, given the available information. To accomplish this, NetFACT employs a novel combination of diagnostic techniques supported by an object-oriented model of the network being managed. This model provides an abstract view of the underlying network of heterogeneous devices. A number of issues were explored in the project including the extensibility of the design to other types of networks, and impact of the practical realities that must be addressed if prototype systems such as NetFACT are to lead to commercial products.
\end{abstract}

\section{INTRODUCTION}

A single fault in a telecommunication network frequently results in a number of alarms being reported to the network operator. This multitude of alarms can easily obscure the real cause of the fault. This phenomena not only increases the skill and time needed to resolve failures, but also increases the probability that one or more failures will be lost in the confusion caused by others. The resulting increase in "mean time to repair" and support center staffing costs make this problem a frequent source of complaints about current network management systems. In order to solve this problem, we must first understand its origins. 
There are a number of reasons why a single fault in a network results in multiple alarms being sent to the network control center. They include:

1. Multiple alarms generated by the same device for a single fault (sometimes known as alarm streaming).

2. The fault is intermittent in nature and each re-occurrence results in the issuance of new alarms.

3. The fault is reported each time a service provided by the failing component is invoked.

4. Multiple components detect (and alarm on) the same condition (e.g., a failing link is detected at both end-points of the link).

5. The fault propagates by causing dependent failures and resultant alarms.

We observe that the first three reasons (above) deal with the same alarm(s) repeated in time, while the last two explain why many different alarms are often triggered by a single fault. With this deeper understanding of the problem, we can now consider solutions.

A variety of solution approaches have been proposed in the literature (Brugnoni(1993), Jordaan(1993), Lor(1993), Sutter(1988)), however, practically deployable, commercial solutions remain elusive. The experiences of the Network Fault and Alarm Correlator and Tester (NetFACT) project, carried out at IBM Research and described in this paper, provide some insight as to why this is the case, and what must be done to overcome the barriers encountered. We divide such barriers into two classes: "basic prerequisites", those things that must be in place before a workable solution can be deployed, and "fundamental technology", the design and algorithms that are needed to solve the problem assuming the basic prerequisites can be put in place. We mention briefly the basic prerequisites and then focus on the fundamental technology issues in the remainder of the paper.

In order for the problem to occur, we can reasonably assume that the most basic of the prerequisites, centralized alarm reporting and storage, is in place. In many cases this may be all the information that is needed to filter out alarms that are repeated in time. Handling different alarms caused by the same fault, however, requires two additional prerequisites: active configuration knowledge (knowledge of the configuration at the time of the failure), and alarm knowledge (knowledge about how the failure condition reported in an alarm from one component relates to other failures in adjacent components of the configuration).

Current technology, such as MAXM(1988), can usually handle the problem of centralized alarm reporting, even from heterogeneous devices using different alarm syntaxes and transport protocols. Standards such as SNMP and CMIP, when fully deployed, will further address the alarm reporting requirements. The problem of acquiring knowledge of the configuration at the time of the failure is somewhat more difficult, but we believe that in most cases this too can be achieved. Active model managers such as RODM (Finkel,1992), that can provide access to sufficiently current representations of the configuration, will help address this need. Alarm knowledge, however, remains an obstacle. We will highlight the requirements in a later section of the paper.

The remainder of the paper discusses the design of the NetFACT system, and our experiences with its development and operation on the Advantis physical backbone network, one of the largest private telecommunications networks in the world. Section 2 provides a overview of the actual algorithms used in the project, section 3 describes the overall system design, and section 4 describes the practical aspects of the problem that had to be accommodated in our design. Section 5 documents some of our observations and conclusions from the project. 


\section{TECHNICAL OVERVIEW}

The approach taken to alarm correlation in NetFACT is to first build a normalized model of the network configuration, normalize the incoming alarms, and then use a generic application to interpret the normalized alarms in the context of the network configuration and prior alarms. This approach stemmed from the observation that three distinct types of knowledge are needed to deduce the underlying faults from the alarms received:

- knowledge about the meaning of the individual alarms,

- knowledge of the network configuration, and

- knowledge of general diagnostic techniques and strategies.

These three types of knowledge would likely come from and, more importantly, be maintained by, separate organizations. Furthermore, alarm knowledge would likely need to be provided and maintained by groups with in depth expert knowledge about the device generating the alarms - this could be many groups, potentially one per type of device. Thus, if the knowledge contained in the system is to be maintainable, it must be partitioned in a way that allows knowledge in any partition to be maintained without awareness or impact to the other partitions. This partitioning is an important and unique aspect of the NetFACT design.

After a brief review of the problem domain in which NetFACT operated, we will describe the diagnostic strategies employed by NetFACT and the representation of the configuration and alarm knowledge required to support those strategies.

\subsection{Domain}

As background information to aid in understanding the diagnostic strategies and configuration models used by NetFACT, we describe briefly the domain of telecommunications networks, in which NetFACT operated. A telecommunication network multiplexes digitized voice and data circuits onto a smaller number of higher speed backbone circuits that carry data between the multiplexers. These higher speed circuits consist of various sequences of "cable" (e.g., wire, fiber, wireless microwave links) and various pieces of equipment (e.g., CSU's, encryptors, repeaters) that in some way transform, monitor, or amplify the physical or logical representation of the data traveling on the circuit. These high speed circuits can themselves be multiplexed onto even higher speed circuits. When data must be transported over long distances, the "cable" used is actually a telephone carrier provided digital circuit (e.g., DS-1, DS-3). We now consider the abstractions used by NetFACT to model telecommunications networks.

\subsection{Configuration Data Model}

The diagnostic process makes use of a normalized model of configuration information to obtain the configuration elements and relationships (connectivity, dependency) between these elements. This model is maintained in an object oriented data base, thus allowing it to be shared with other network management applications. In keeping with our objective to make NetFACT sufficiently general to support other types of networks, the normalized model is somewhat more abstract than the description of telecommunications networks given in the previous section. As a result, a given piece of equipment may be represented by more than one object in the model.

In the NetFACT data model, network components are classified as paths, nodes, or shared resources. The normalized data model has one further level of detail (i.e., types of paths, nodes, etc), but this will not be discussed here due to space constraints. A path is defined to be a connection between end points, over which the same data flows. A node is 
a network component that in some way processes the data flowing over a path. Paths may contain nodes and other paths. A node with one connection to a path is called an end point of that path. All nodes that are not endpoints have exactly two connections. Nodes may depend on one or more shared resources, each of which may also depend on one or more shared resources. A given shared resource may support multiple nodes/shared resources, thus dependency is a many-to-many relationship. To apply this model to telecommunication networks, we use paths to represent both the circuits and "cables" in the network, while nodes are used to model the various pieces of telecommunications equipment on a circuit, including the interface cards in the multiplexors that are the endpoints of the circuits. A complex device with many ports, such as a multiplexor, is modeled as a collection of nodes (representing interfaces) that are dependent upon a common shared resource (representing the common elements of the device such as the power supply, backplane, and control circuitry). More elaborate models can be constructed, if needed.

The normalized relationships modeled by NetFACT include data-flow, composition, and dependency. Data flow and dependency are used to follow the potential propagation of faults, while composition is used to help optimize the diagnostic algorithms by reducing the portion of the network that they must explore in certain situations.

Inheriting from the normalized model are the sub-classes that are unique to each component type. These classes contain any attributes or methods that are needed to convert the alarms for a specific type of device into the normalized form. It is these device type specific classes that are instantiated with the network configuration.

The picture in Figure 1 shows the class instances and relationships that NetFACT uses to model a typical telecommunications circuit. The circuit begins, in the upper left corner, with an IDNX trunk card (N020C050) connected to another IDNX trunk card (N111C000) via a DS1 path (IBM-002003). The DS1 path object does not represent any single physical object but rather the sequence of objects it contains (indicated with the dashed lines). Thus, data flows from the IDNX trunk card (N020C050) through an encryptor (00004553), and then through a CSU (TC006480). At this point the circuit is multiplexed onto a DS3 path (IBM-17958). The use of this DS3 by the DS1 (IBM-002003) is represented by the DS3_channel object (G002); this allows us to follow the original data flow through the DS3 and locate it on the far side. The pair of Network_ports (G006,G003) are used to represent the portion of the circuit provided by a common carrier. Note that the data enters the carrier's network on a DS3 (G006), is demultiplexed by a multiplexor not visible to NetFACT, and exits the carriers network as a DS1 (G003). After exiting the carrier's network, the data flow proceeds thru the CSU (TC0000008), the encryptor (00000004), and finally to the IDNX_Trunk (N111C000) which is the end of the circuit. The multiplexors that are visible to NetFACT are represented by a combination of node objects (e.g., IDNX_Trunk, M13_T1_port) and shared resources (e.g., IDNX_box, M13_box).

In addition to configuration data (i.e., object identity, type, and relationships), the data model also includes real time component status information that is both used and updated in the process of building the normalized alarm representation.

\subsection{Diagnostic Strategies}

In general, the approach to diagnosis taken in NetFACT is to employ a collection of techniques specialized to the type of topology that encompasses the fault/alarms being considered. Note that we specialize to the type of topology and not to the type of device as is more commonly done in rule based expert systems.

In the current implementation of NetFACT, two diagnostic techniques are used. The first, which we call path analysis, handles problems relating to the failure of a path. Typically, one component of the path (either a piece of equipment or a carrier circuit) fails and all communications over the path are stopped. Various components on the path report the failure by generating alarms. Path analysis processes these alarms and determines which 


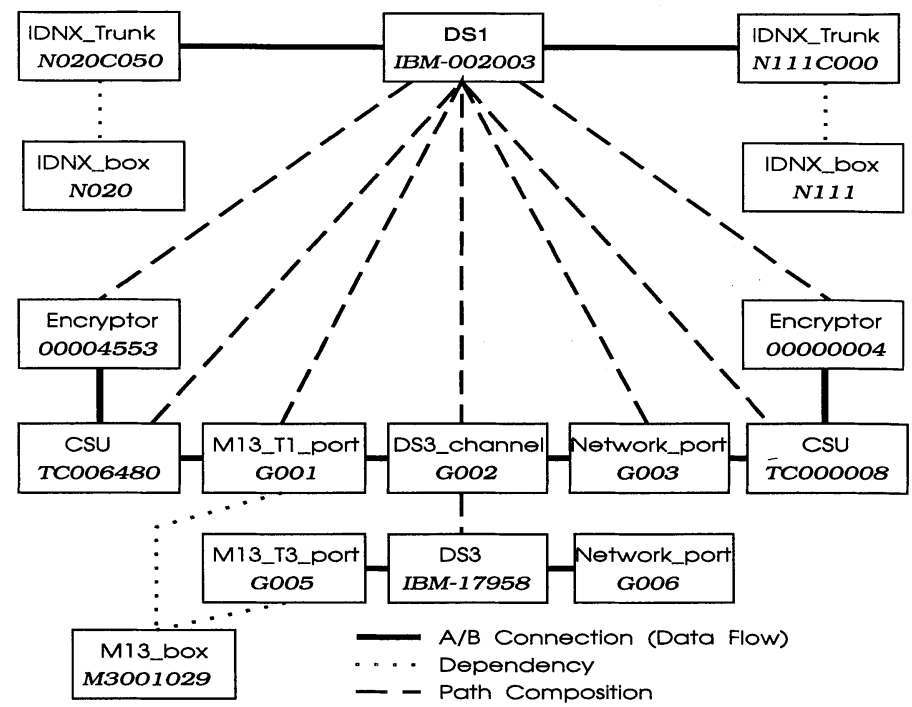

Figure 1. Example of NetFACT Data Model.

components on the path are most likely to be responsible for the failure. Once this determination has been made, the second technique, which we call tree search, is used to determine whether the nodes (or sub-paths) identified are responsible for the failure themselves, or whether they are failing because of a problem in components on which they are dependent (e.g., shared resources). Looking at the relationships shown in Figure 1, path analysis locates failures that propagate along horizontal relationships (i.e., data flow), while tree search locates failures that propagate along vertical relationships (i.e., dependency).

Path analysis employs a voting technique to sum all the evidence contained in the alarms received from the nodes on a given path. Each normalized alarm provides an indication of where the cause of the problem might lie, relative to the node reporting the alarm. Possible locations include the node itself, a matching peer device, and somewhere in either direction of data flow. The likelihood that the cause of problem is in any of these possible locations is expressed as an integral number of votes for each possible location. This allows the alarm to express some degree of uncertainty about the precise location of the source of the problem it is reporting. For example, if a CSU detects a problem on its line side, the normalized alarm generated as a result would contain one vote for the device itself (it is always possible that the problem is in the line interface of the CSU) and two votes for all devices on the path in the direction of the line; devices in the direction of the DTE equipment would receive no votes. The votes are summed for each component of the path and the components with the most votes or second most votes are explored using the tree search technique.

Tree search explores the dependency tree for a given component to determine if a lower level problem is causing the component to fail. The exploration process considers both direct evidence (i.e., alarms) and indirect evidence (i.e., how many users of a lower level component are experiencing difficulties.) Indirect evidence must be used because failing components do not always generate alarms. In cases where the failure of a given component or path could be caused by " $n$ " different lower level component failures, for 
each of which only indirect evidence exists, heuristics are used to choose the component most likely to be the cause of the failure. Diagnostic tests, if available, could also be used to help resolve such ambiguities. If the lower level resource suspected of failing is a path (such as a DS-3), path analysis is invoked recursively.

As the above diagnostic strategies proceed, previously independent problems/alarms are causally related and the overall number of "open" problems is reduced. After a set amount of time, a problem that cannot be related to another is surfaced to the operators through a user interface application. In general, problems in NetFACT are moved through phases (states) of a problem lifecycle. Ignoring some complexities that will be discussed in a later section of the paper, the basic problem lifecycle in NetFACT involves the following states:

Awareness Build internal representation of the alarm and wait briefly for additional related alarms to arrive

Get config Obtains relevant configuration from the configuration model

Diagnosis Use the diagnostic strategies to identify the cause of the alarm

Recovery Await the recovery of the network components impacted by the problem

Closure Mark the problem as closed and direct any further alarms from the components impacted to open new problems

Purge An operator purges the problem from the system

Figure 2, together with the explanation below, shows how the diagnostic techniques are applied to locate the root cause of a problem. The sequence of events is as follows:

1. Components $\mathrm{A}, \mathrm{B}$, and $\mathrm{E}$ send alarms (The alarm notation shows the number of votes for self inside the circle and the number of votes in each direction of data flow at the ends of the directional arrows.)

2. Path analysis first applies the relative voting information in the alarms to the path configuration

3. Path analysis then sums the votes for each component in the configuration and determines that components $\mathrm{C}$ and $\mathrm{D}$ are the most likely causes of the path failure; components $\mathrm{B}$ and $\mathrm{E}$ are second choices

4. Tree search is invoked; only component $D$ is found to have a dependency: it is dependent on component $F$

5. Components $\mathrm{X}, \mathrm{Y}$, and $\mathrm{D}$ are all users of component $\mathrm{F}$, but each is on a different path; the paths containing components $\mathrm{X}$ and $\mathrm{Y}$ are also experiencing failures (not shown)

6. Components $\mathrm{X}$ and $\mathrm{Y}$ are also prime suspects in their respective path failures (not shown); tree search will identify component $F$ as the most likely cause of failures of the paths containing components $\mathrm{X}, \mathrm{Y}$, and $\mathrm{D}$

7. NetFACT will open a single problem with component $F$ as the most likely cause.

\subsection{Alarm Normalization}

Alarms are received in a variety of syntaxes and must be translated to a normalized form with consistent syntax and semantics. The information contained in the normalized alarm includes:

- The identity of the object to which the alarm refers

- The impact to the behavior of the object (e.g., UP, DOWN)

- Votes representing the likely source of the problem that caused the alarm

- Miscellaneous information such as timestamps and alarm id

When an alarm is received, the corresponding model object is located in the data base and used to determine if the alarm contains new information not reported by previous alarms. Alarms containing new information are normalized and passed to the diagnostic application; the status information in the object model is updated in the process. 


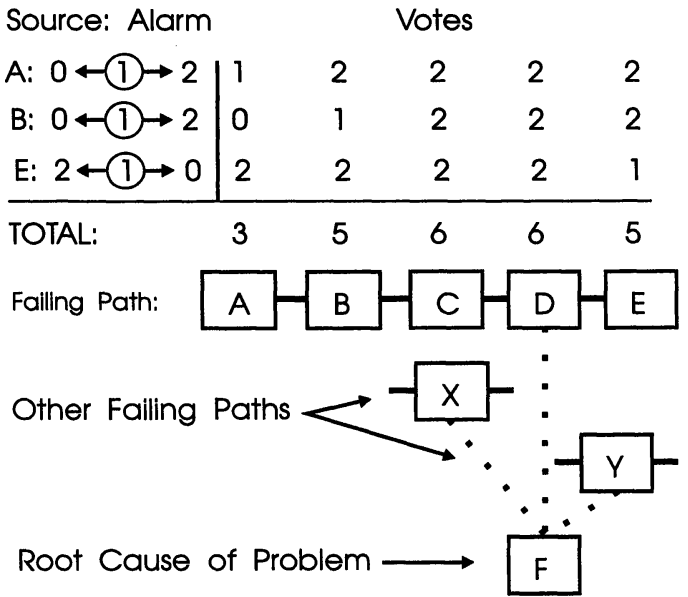

Figure 2. Diagnostic/Correlation Example (see text for explanation).

\section{SYSTEM DESIGN}

The diagram in Figure 3 shows the components of the NetFACT system and the data flows between them. The system was implemented on an MVS/390 system using IBM's NetView network management system. This allowed the configuration model to be implemented using NetView's Resource Object Data Manager (RODM), a high performance, object oriented data manager (Finkel,1992).

The NetFACT components (Figure 3) are best understood by following the processes in which they participate. NetFACT has a configuration model update process and an alarm handling process. The configuration model update process extracts the current version of the configuration from a number of different tables in an SQL database, and updates the object data model (in RODM) to this version of the configuration. This is accomplished without impacting the availability of the alarm handling process, or other applications that may also be using RODM.

The alarm handling process begins with the receipt of an alarm from the network. NetView's alert automation facilities then select and dispatch the appropriate command procedure (script) to generate the normalized form of the alarm. In the process of doing this, the command procedure locates the corresponding object in RODM and updates its status accordingly. If the alarm contains information that is important to the diagnostic algorithms (and has not been previously reported), it is passed through RODM to the NetFACT application. Here it is operated upon by the diagnostic procedures described in the previous section. If a new problem is identified, an object is created in RODM to represent the problem. The operator interface component can query these objects and display information about the faults they represent to a human operator. In addition, the creation of the problem object can cause a problem record to be opened in a problem management system, such as IBM's INFO/MGT product. 


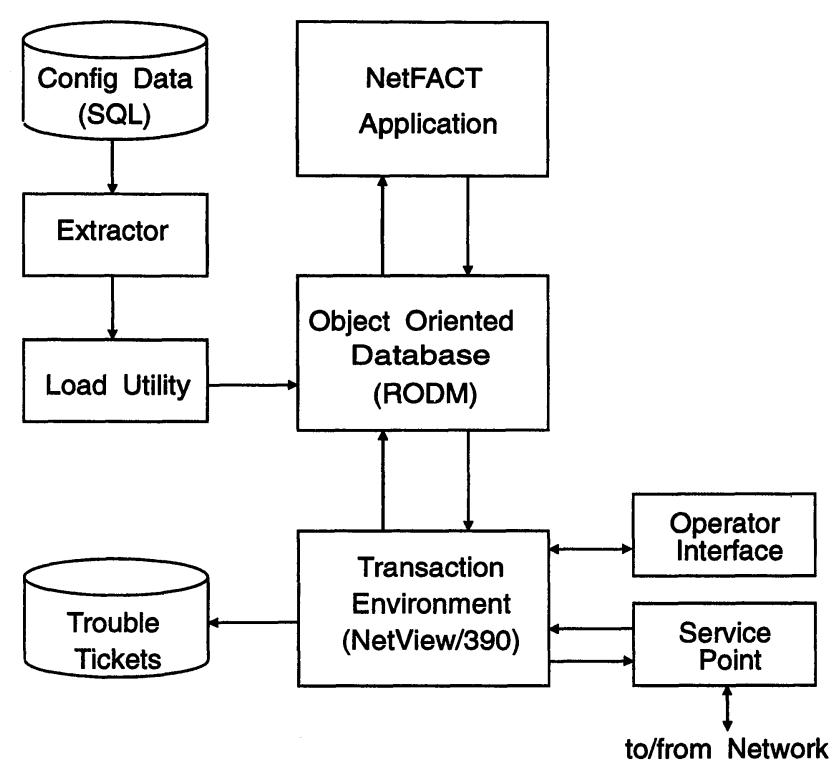

Figure 3. NetFACT System Design.

\section{PRACTICAL CONSIDERATIONS}

In the process of developing NetFACT and testing it with a real alarm stream from the Advantis physical network, a number of practical problems were encountered. Many of these were solved during the course of the development and we continue to study those that were not. We discuss some of those problems here along with other observations made during the project.

\subsection{Noise}

The first practical reality that we encountered was "noise". In the ideal case, a problem detected by a component results in one alarm to indicate that the problem has been detected, and another to indicate that the problem has been resolved and correct behavior restored. Some problems do, in fact, result in such clean notifications - unfortunately, many others do not.

We refer to alarms we wish we didn't have to process as "noise" and group them into the six categories shown in Figure 4. The taxonomy is useful because it allows NetFACT to employ different strategies to deal with different kinds of noise.

Alarms that to do not usually indicate a problem with the behavior of the component, although they may help explain a problem reported by other alarms, are classified as insignificant information. The information may optionally be retained in the component's object model, where it can be used in answering specific queries that NetFACT may direct at the object model. Redundant information and streaming alarms can be filtered out with the 


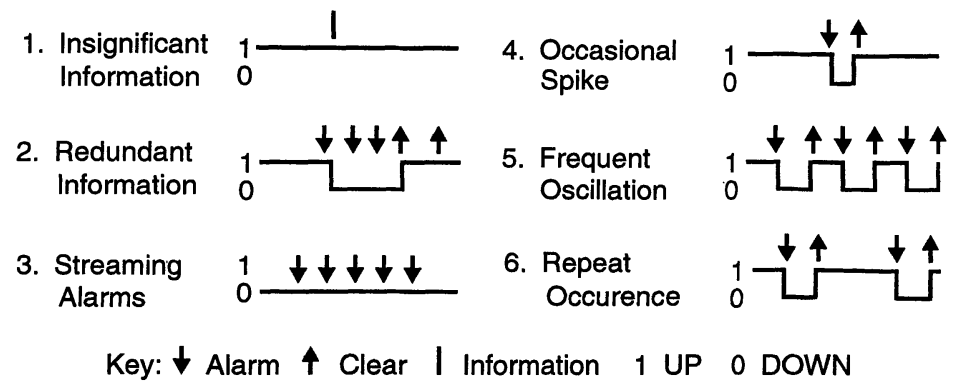

Figure 4. Categories of Noise.

help of the state information kept in the object model. If necessary, streaming alarms can be filtered closer to the source, to avoid the overhead of transmitting them to the central site. The occasional spike is suppressed by extending the problem lifecycle to include a verification stage, where a problem must persist for a specified, but short, period of time before NetFACT will process it. Likewise, frequent oscillations are suppressed by requiring that problems remain resolved for a period of time before they are allowed to enter the closed stage of the lifecycle. Repeat occurrences of a problem occur with sufficient separation that re-diagnosis is appropriate. An automated link from the repeat occurrences back to the original problem was not implemented, although it seems feasible.

\subsection{Hidden Dependencies}

Many of the systems described in the diagnosis literature, as well as earlier versions of NetFACT, are aimed at the goal of finding a minimum number of faults that will explain the observed symptoms. Since faults occur relatively infrequently, it seems reasonable that the probability of multiple faults occurring simultaneously is extremely low. Thus the heuristic that the correct root cause of a number of related symptoms is likely to be a minimum number of faults. In the real world, this is probably true.

In the world of NetFACT, however, visibility is often limited and not all the dependencies that would point to the single root cause of a set of symptoms are known (Figure 5.) The common cause of a set of path outages may be within a carrier's network. Simply applying the above heuristic may result in incorrectly identifying a common node that is visible to NetFACT as the cause, when really the correct cause is a hidden common element inside the carrier network. To address this problem, NetFACT reports such failures as independent faults. (A possible enhancement would be to group independent failures that occur within the same carrier network at nearly the same time, to suggest a probable correlation.)

This problem helped persuade us to change our basic strategy of how NetFACT should correlate alarms. We changed from using a global optimization strategy based on the "minimum number of faults" heuristic, to a strategy of finding the "best explanation for each symptom". Global knowledge is still used in determining the cause of a symptom, but it is considered weaker evidence than actual alarms, and no overall global optimization is attempted. When a high percentage of users of a resource are experiencing a failure, this suggests that the supporting resource may be responsible. Each user of the resource is 


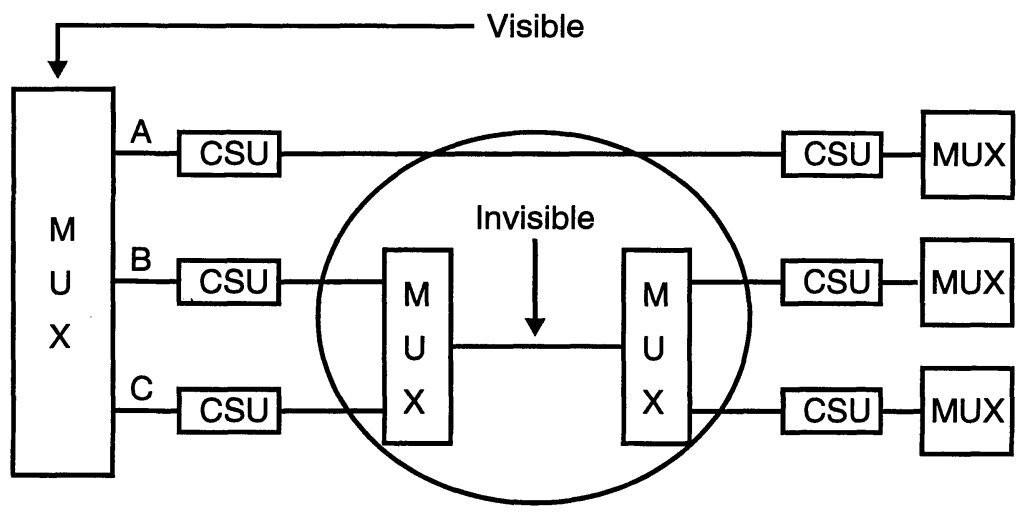

Figure 5. Example Configuration with Hidden Dependencies.

required to believe that the shared resource is the most likely cause of the problem, however, before the association can be made.

\subsection{Complex Dependencies}

The next problem we encountered involved the nature of the dependency relationships between network components. NetFACT supports only simple dependency relationships, where the dependent resources depend solely on the binary availability of the supporting resources. When a supporting resource fails, all dependents fail with it (and nearly simultaneously). Unfortunately, not all dependencies are simple.

Some resources depend on logical combinations of other resources. For example, the availability of an SNA transmission group depends on at least one of the link in the group being active.

Other resources depend instead, on a quantitative amount of capacity in the shared resource. In these cases, a sudden drop in capacity can cause failures in the dependent resources around the time of the capacity drop. A later failure of a dependent resource is much less likely to be explained by the capacity drop (unless the failing resource has requested additional capacity.) An example of this class of problem is the allocation of capacity on IDNX CPU cards needed to support DS-1 circuits connecting IDNX nodes. When a CPU card fails in a node (multiplexor) without sufficient backup CPU capacity, a number of DS-1 trunks connected to that node are dropped. A related class of problems, involving buffer pools, can be found in packet switches.

\subsection{Missing Data}

NetFACT relies totally on unsolicited alarms to get its information about what is happening in the network. This limitation was project related and stemmed mainly from the technical difficulties of interconnecting the various network management systems being used at 
Advantis, in addition to concerns about the potential of NetFACT interfering with production operations. This limitation proved to be a serious problem. Unsolicited data alone does not always result in a complete or even accurate picture of what is happening in the network. Scenarios involving missing alarms or status updates include:

- Data received from only one end of a path, or one of a pair of matched devices

- No indication that a given device has recovered

- No path to receive data from a remote device

State data derived from alarms and unsolicited status updates must be treated carefully in light of the above. The NetFACT system associates a time stamp with each state of each resource in the state model. This information is very useful when viewing or analyzing resource state information.

It is important to note that if NetFACT were able to solicit status information from the network components, it would be able to use its knowledge of the network status and problems to reduce the number of solicitations needed. Conventional timer driven polling applications would not have such knowledge and therefore would be less efficient at collecting status information. Thus, NetFACT's powerful knowledge base has interesting implications for the overall design of network management systems.

\subsection{Implementation}

We are often asked about the programming languages and tools used to implement NetFACT. The diagnostic application is written in ANSI C, rather than a rule based language. While there were times when a rule based approach seemed more desirable, we still believe that, overall, the procedural approach resulted in a more robust and maintainable application. $\mathrm{C}++$, had it been available then in the MVS environment, would have resulted in somewhat more maintainable code.

The RODM data store proved quite adequate for our data modeling needs. Both its execution speed and object oriented capabilities greatly facilitated our implementation.

\section{OBSERVATIONS, ASSESSMENTS, AND CONCLUSIONS}

We now return to the question of what must be done to make systems such as NetFACT into practical, commercial products. Based on our observations, probably the greatest need is to bring together the development of alarm reporting standards, configuration models, and diagnosis/correlation algorithms. By bringing together these currently independent activities, we will be able to insure that the incoming alarms can be understood in terms of the configuration model and that they will contain sufficient information to drive the correlation algorithms. Until adequate alarm reporting standards are in place, systems such as NetFACT will be forced to translate the individual alarms into a suitable normalized form. This translation typically requires knowledge about the semantics of each individual alarm. We believe the discovery and maintenance of this information is both difficult and costly and thus will be a significant impediment to vendors wishing to bring commercial products to market, in anything but a very limited context.

We remain satisfied with the overall system design used in the NetFACT project and we believe it can serve as a model for future implementations. The power of the locally cached object oriented network model can be more fully exploited once solicited diagnostic testing of network components is introduced.

Additional diagnostic/correlation techniques will be needed to support other types of networks such as packet routing networks (IP). How new diagnostic techniques can be easily incorporated into the existing algorithm remains an interesting area for future research. Despite the apparent complexity of such an approach, we remain convinced that 
a collection of diagnostic/correlation techniques is likely to outperform any single unified approach. In addition, using knowledge of past experience in the diagnostic algorithms offers interesting possibilities. Historical knowledge would be useful in optimizing the search for the cause of a failure, in detecting repeat occurrences of problems, and in recommending a course of action once the cause of a fault has been determined.

Finally we would like to emphasize the importance, benefits, and difficulties of conducting experiments on a real network. In our early work on NetFACT, a small 2-3 node test network was used to collect the alarms resulting from manually induced failures (unplugging various cables). Unfortunately the data collected from these experiments was not indicative of the full magnitude and scope, and especially the interaction, of problems we later encountered running with the full Advantis network. In particular, the problems relating to noise, missing data, and the interaction between multiple faults occurring in the same period of time were not anticipated based on experiences with the "test network" faults. On the down side, the project experienced considerable delays while waiting for various instrumentation and network management connectivity problems to be resolved.

While NetFACT is far from the ideal envisioned by many network managers, it represents an important step toward achieving the goal of developing a global automated system for problem management. The NetFACT project has shown that practical solutions to the problem of alarm correlation are possible, although additional work is necessary, especially in the area of alarm standardization, before such solutions are likely to become commercially available. Furthermore, we believe that our design shows how the vast amount of knowledge used by such a system can be organized and partitioned in a way that will allow it to be easily maintained.

\section{REFERENCES}

Brugnoni, S., et al. (1993) An Expert System for Real Time Fault Diagnosis of the Italian Telecommunications Network, in Proceedings of the International Symposium on Integrated Network Management III (ed. H.-G. Hegering and Y. Yemini), IFIP, San Francisco, CA

Finkel, A. and Calo, S.B. (1992) RODM: A Control Information Base. IBM Systems Journal, V31 N2,252-269

Jordaan, J. F. and Paterok, M. E. (1993) Event Correlation in Heterogeneous Networks Using the OSI Management Framework, in Proceedings of the International Symposium on Integrated Network Management III (ed. H.-G. Hegering and Y. Yemini), IFIP, San Francisco, CA

Lor, K.-W. E. (1993) A Network Diagnostic Expert System for Acculink(tm) Multiplexers, in Proceedings of the International Symposium on Integrated Network Management III (ed. H.-G. Hegering and Y. Yemini), IFIP, San Francisco, CA

MAXM Corp. (1992) MAXM System Administrator's Guide, International Telecommunications Management, Inc., Vienna, VA

Sutter, M. T. and Zeldin, P. E. (1988) Designing Expert Systems for Real-Time Diagnosis of Self-Correcting Networks, IEEE Network Magazine,September 1988,43-51 